\title{
Energy Management Strategies for a Pneumatic-Hybrid Engine Based on Sliding Window Pattern Recognition
}

\author{
A. Ivanco, G. Colin, Y. Chamaillard*, A. Charlet and P. Higelin \\ Institut PRISME/EPM, Université d'Orléans, 8 rue Léonard de Vinci, 45072 Orléans Cedex 2 - France \\ e-mail: andrej.ivanco@univ-orleans.fr - guillaume.colin@univ-orleans.fr -yann.chamaillard@univ-orleans.fr \\ alain.charlet@univ-orleans.fr-pascal.higelin@univ-orleans.fr \\ * Corresponding author
}

\begin{abstract}
Résumé - Stratégies de gestion de l'énergie pour un moteur hybride pneumatique basées sur la reconnaissance du cycle de conduite - Cet article présente comparativement des stratégies de gestion de l'énergie pour un nouveau concept de moteur hybride : l'hybride pneumatique. Dans cette configuration spécifique, c'est le moteur lui-même qui est hybridé (et non le véhicule). Plusieurs stratégies de gestion d'énergie sont proposées dans cet article. La première est dite Causale (CS) car basée sur des principes heuristiques de décision. La deuxième est basée sur la minimisation d'un critère d'équivalence et est appelée stratégie à Coefficient de Pénalité Constant (CPC). Dans ce cas, les flux d'énergie (depuis chaque source chimique ou pneumatique) sont décrits dans des unités identiques. Ainsi, pour un même travail à produire, il est possible de faire une «balance» entre la consommation nécessaire selon chacune des deux sources d'énergie, et ceci avec un coefficient de pondération constant. La troisième stratégie utilise un coefficient de pondération variable selon la quantité d'air disponible dans le réservoir (i.e. état de charge) et est appelée stratégie à Coefficient de Pénalité Variable (VPC). Dans ce cas, le coefficient de pénalité est une fonction non-linéaire de la pression dans le réservoir. Un autre raisonnement consiste à considérer qu'il est nécessaire d'adapter également le coefficient à la situation de conduite (embouteillage, urbain, routier, autoroutier...), pour cela il est impératif de reconnaître la situation de conduite. Le coefficient peut alors être adapté, selon la situation reconnue à la valeur optimale prédéterminée pour des situations types. Cette stratégie, à reconnaissance de situation de conduite (DPR), se base sur une fenêtre glissante où la situation de conduite est considérée à changements lents (conservatisme). Une partie du travail a été d'optimiser la taille de la fenêtre d'identification. Les situations de conduite types sont décrites par des modèles statistiques (densité de présence). La reconnaissance du cycle est basée sur une fonction de corrélation. Afin de comparer les résultats obtenus sur différents cycles de conduite (homologués et Artemis) avec les différentes stratégies proposées, les consommations minimales atteignables obtenues par Programmation Dynamique (DP) sont également données. Les résultats montrent que $40 \%$ de gain de consommation peuvent être atteints sur certains cycles. Les résultats obtenus avec les stratégies «adaptatives» (VPC et DPR) sont meilleurs que ceux obtenus avec les stratégies «constantes» (CS et CPC). De plus, les résultats obtenus sont proches des résultats optimaux obtenus avec la programmation dynamique.
\end{abstract}

Abstract - Energy Management Strategies for a Pneumatic-Hybrid Engine Based on Sliding Window Pattern Recognition - This paper presents energy management strategies for a new hybrid pneumatic engine concept which is specific by its configuration in that it is not the vehicle but only the 
engine itself which is hybridized. Different energy management strategies are proposed in this paper. The first is called Causal Strategy (CS) and implements a rule-based control technique. The second strategy, called Constant Penalty Coefficient (CPC), is based on the minimization of equivalent consumption, where the use of each energy source is formulated in a comparative unit. The balance between the consumption of different energy sources (chemical or pneumatic) is achieved by the introduction of an equivalence factor. The third strategy is called Variable Penalty Coefficient (VPC). In fact, it is beneficial to consider the equivalence coefficient as variable within the amount of pneumatic energy stored in the air-tank i.e. state of charge, because the choice of propulsion mode should be different if the tank is full or empty. In this case, the penalty coefficient appears as a non linear function of the air-tank state of charge. Another way to adapt the penalty coefficient is to recognize a reference pattern during the driving cycle. The coefficient value can then be changed according to an optimized value found for each of the reference cycles. This strategy is called Driving Pattern Recognition (DPR). It involves a technique of sliding window pattern recognition. The concept is to convert the whole driving cycle into smaller pieces to which the equivalence factor can be appropriately adapted. This strategy is based on the assumption that the current driving situation does not change rapidly and thus the pattern is likely to continue into the near future. The identification window size is a parameter which has to be adjusted to attain the maximum of identification success over the reference cycle. We propose to define reference patterns as statistical models. The pattern recognition method is based on a correlation function. To improve analysis, all the results obtained with different energy management strategies are compared with a Dynamic Programming approach (DP) considered as the optimal solution. Results show that about $40 \%$ of fuel saving can be achieved by DP. The VPC and DPR strategies give better results than the CPC strategy, not so far from the results obtained with DP.

\section{DEFINITIONS, ACRONYMS, ABBREVIATIONS}

CPC
CS
CVO, CVC
Cons.
DP
EVO, EVC
ICE
IVO, IVC
NEDC
PC
PHE
TDC
SOC
VPC

Constant Penalty Coefficient

Causal Strategy

Charging Valve Opening/ Closure

Consumption

Dynamic Programming

Exhaust Valve Opening/ Closure

Internal Combustion Engine

Intake Valve Opening/ Closure

New European Driving Cycle

Penalty Coefficient

Pneumatic Hybrid Engine

Top Dead Center

State Of Charge

Variable Penalty Coefficient

\section{SYMBOLS}

$C_{p} \quad$ Specific heat constant pressure

$k \quad$ Discrete time

$L C V \quad$ Lower Calorific Value

$l_{d} \quad$ Developed length

$m \quad$ Mass

$n \quad$ Power of the SoC term

$N_{e} \quad$ Engine speed

$p_{t} \quad$ Air-tank pressure
$(\mathrm{J} /(\mathrm{kg} \cdot \mathrm{K}))$

$(-)$

$(\mathrm{J} / \mathrm{kg})$

$(\mathrm{m} / \mathrm{tr})$

(kg)

$(-)$

(tr/min)

(Pa)

$\begin{array}{lll}r & \text { Specific gas constant } & (\mathrm{J} /(\mathrm{kg} \cdot \mathrm{K})) \\ T_{e} & \text { Engine torque } & (\mathrm{N} / \mathrm{m}) \\ T_{t} & \text { Tank temperature } & (\mathrm{K}) \\ U & \text { Internal energy } & (\mathrm{J}) \\ V_{d} & \text { Desired speed } & (\mathrm{m} / \mathrm{s}) \\ W & \text { Work } & (\mathrm{J}) \\ \gamma & \text { Acceleration } & \left(\mathrm{m} / \mathrm{s}^{2}\right)\end{array}$

\section{INTRODUCTION}

Several concepts have been proposed by engineers worldwide in order to improve global powertrain efficiency (including the Internal Combustion Engine (ICE)) and consequently to reduce the environmental impact. The hybrid pneumatic engine is one such concept and differs significantly from the current focus on a hybrid-electric configuration in using compressed air instead of electrical power.

The concept of the hybrid-pneumatic engine [1, 2] is specific in that it is not the vehicle but the engine itself which is hybridized. It is based on the traditional ICE, with a builtin pneumatic motor representing a supplementary pipe linking the combustion chamber (Fig. 1 - Position 2) to the compressed air tank (Fig. 1 - Position 3) through an additional charging valve (Fig. 1 - Position 1). Thanks to this modification the engine can store kinetic energy from the vehicle to the air tank without the need for an additional compressor. Conversely, the stored energy can be used to produce torque (i.e. the ICE is used as a pneumatic motor). 


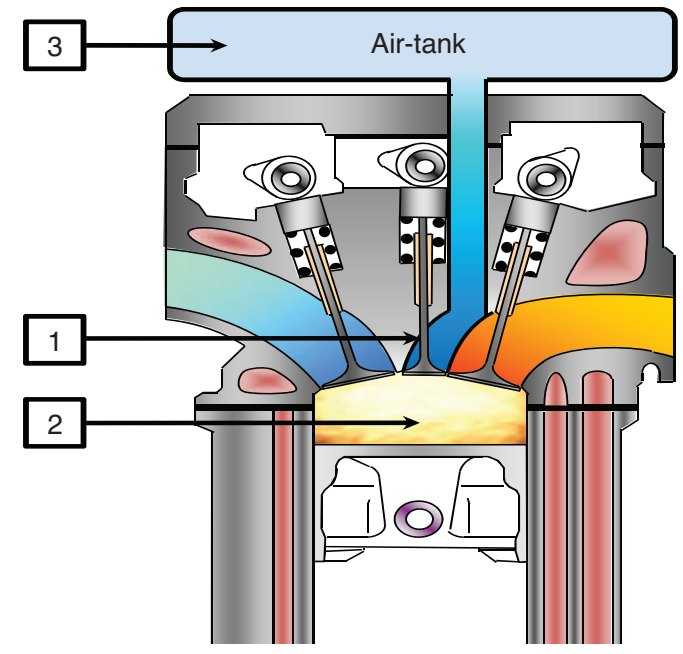

Figure 1

Pneumatic-hybrid scheme.

The pneumatic-hybrid engine can be considered as a parallel hybrid configuration, when both the ICE and the pneumatic motor are coupled on a single crankshaft and can work separately or together in a supercharging mode.

This concept has three main advantages:

- the ability to recuperate kinetic energy;

- no need for an additional clutch, reducer or continuously variable transmission;

- erasing turbo lag during acceleration thus offering a significant downsizing potential.

Simulation results achieved with different driving cycles, vehicles and energy management strategies show that, with this configuration, interesting performance in terms of fuel saving can be obtained.

To take advantage of the proposed concept it is necessary to define and optimize not only the air-tank size or dimension of the charging valve, but also the energy management strategies.

The objective of this paper is to propose, adapt and compare different energy management strategies for the concept of the hybrid-pneumatic engine. In a simulation environment, the strategies are compared for different driving cycles.

\section{SYSTEM MODELLING}

The request of vehicle speed, followed by a virtual and perfect driver, is defined by the driving cycles.

\subsection{Driving Cycles}

To evaluate the system performance, six different driving cycles are used. The first is the NEDC (New European Driving
Cycle, Fig. 2) which is a standard for fuel consumption evaluation. Four other driving cycles [3] represent a more realistic overview of driving situations (Traffic-jam, Urban, Road, and Highway). The last one called reference cycle consists of sequence of Artemis cycles.

\subsection{Vehicle Model}

In this paper, a simple model of a middle class vehicle (Vehicle mass $m$ of $1500 \mathrm{~kg}, 5$ speed gear box and 1.61 gasoline engine) is used. A discrete model is considered with a sampling period $\Delta t$ of $0.5 \mathrm{~s}$. To enable comparison between the energy management strategies of the hybrid and non-hybrid configurations, the gearbox switching strategy is always coherent with that defined in the conventional engine mode.

Taking into account the vehicle model and power transmission, the cycle speed $v_{d}$ is interpreted as a torque demand to be satisfied by the engine $T_{e}$.

The driver receives the desired speed signal $v_{d}(k)$ which is compared to the previous speed $v_{d}(k-1)$ in order to get the acceleration:

$$
\gamma(k)=\frac{v_{d}(k)-v_{d}(k-1)}{\Delta t}
$$

Considering the forces acting on the vehicle (aerodynamics $F_{a}$, rolling resistance $F_{r}$ ), we have:

$$
m \cdot \gamma(k)=-F_{a}(k)-F_{r}(k)+F_{t r}(k)
$$

from which the traction force $F_{t r}$ is calculated. The transmission chain characteristics (final reduction ratio $\rho_{a}$ and gear-box ratio $\rho_{g b}$ ) yield the engine torque $T_{e}$ to be produced (shown in Fig. 2).

$$
T_{e}(k)=\frac{F_{t r}(k) \cdot \rho_{g b} \cdot \rho_{a} \cdot l_{d}}{2 \pi}
$$

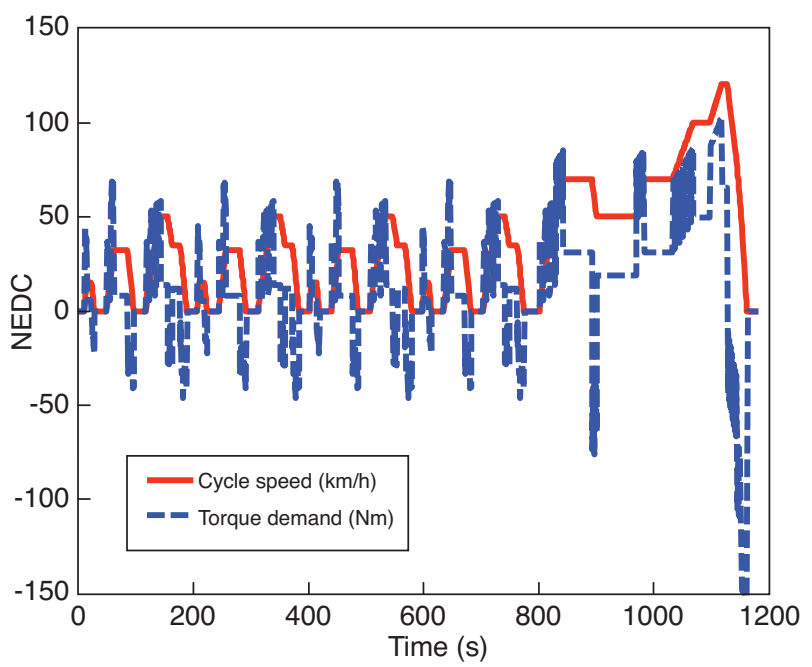

Figure 2

NEDC driving cycle. 


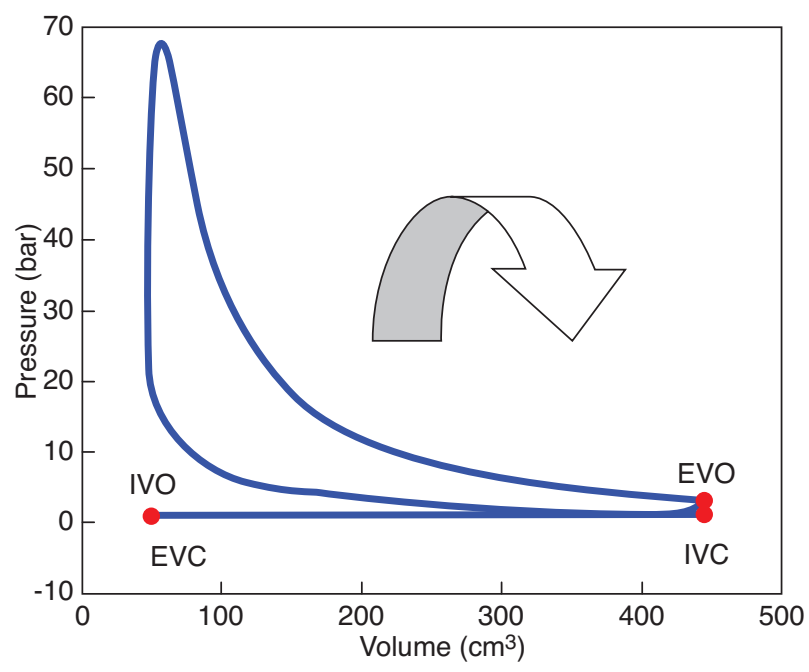

Figure 3

Conventional mode indicator diagram.

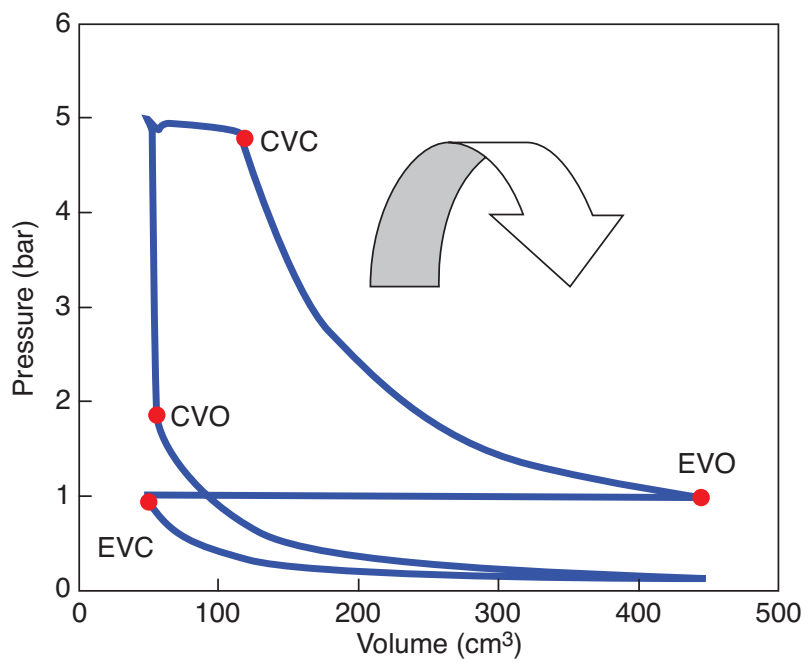

Figure 4

Pneumatic mode indicator diagram.
The control of torque production is divided into two levels. A supervisory level runs the algorithm of choosing the propulsion mode in order to optimize fuel consumption. The low-level controller tunes the engine parameters to produce the requested torque. A basic overview of the propulsion modes is given below with indicator diagrams.

Some physical limitations are taken into account.

For the idle consideration, the conventional mode cannot be used if the engine speed $N_{e}$ is under the idle speed $N_{\text {idle }}$ :

$$
N_{e} \geq N_{\text {idle }}
$$

Another restriction is for the engine torque $T_{e}$, which should be kept within the limits for maximum and minimum production capacity.

$$
T_{\text {conv_min }}(N) \leq T_{e} \leq T_{\text {conv_max }}\left(N_{e}\right)
$$

where $T_{\text {conv_max }}$ (resp. $T_{\text {conv_min }}$ ) is the maximum (resp. minimum) conventional engine torque.

Regarding the pneumatic mode, if the air-tank pressure $p_{t}$ is below a minimum threshold value 5 bar, pneumatic Stop\&Start cannot be used. On the other hand a maximum pressure of approximately 25 bar can be achieved, which depends on the compression ratio of the engine, whereupon the pumping mode quickly loses its effectiveness.

To reduce calculation time, all the thermodynamic cycles have been synthesized into the cartography form [4]. The representativeness of the quasi-static model of the engine consumption has been derived from test bench measurements.

To model the air tank pressure $p_{t}$, the perfect gas law is used:

$$
p_{t}=\frac{m_{t} \cdot r \cdot T_{t}}{V_{t}}
$$

where $m_{t}$ is the tank air mass, $r$ is the specific gas constant, $V_{t}$ is the tank volume of $50 \mathrm{~L}$ and $T_{t}$ is the tank temperature:

$$
T_{t}=T_{a m b} \cdot\left(\frac{p_{t}}{p_{a m b}}\right)^{\frac{r}{C_{p}}}
$$

where $T_{a m b}$ is the ambient temperature and $p_{a m b}$ ambient pressure and $C_{p}$ is specific heat capacity at constant pressure.

\subsection{Pneumatic-Hybrid Driving Modes}

Altogether, four driving modes are possible:

- two propulsive modes: conventional and pneumatic;

- one recuperative mode: pneumatic pump;

- and one alternative engine stop mode.

\subsubsection{Conventional Mode}

The Conventional cycle (Fig. 3) is a classical four-stroke combustion cycle (intake, compression, expansion and exhaust) the effective work output is adjusted by air mass flow through the throttle.

\subsubsection{Pneumatic Mode}

The pneumatic mode is used to produce torque from compressed air and is able to do so even from the zero engine speed. In this mode the intake phase is suspended. The cycle (Fig. 4) begins at Top Dead Center (TDC) with Charging Valve Opening (CVO) then the in-cylinder pressure increases with the mass of air flowing from the air tank to the engine. The indicated work in this cycle can be continuously controlled by CVO and Charging Valve Closure (CVC). Basically, $\mathrm{CVO}$ is adopted to take place just after reaching TDC. Then 


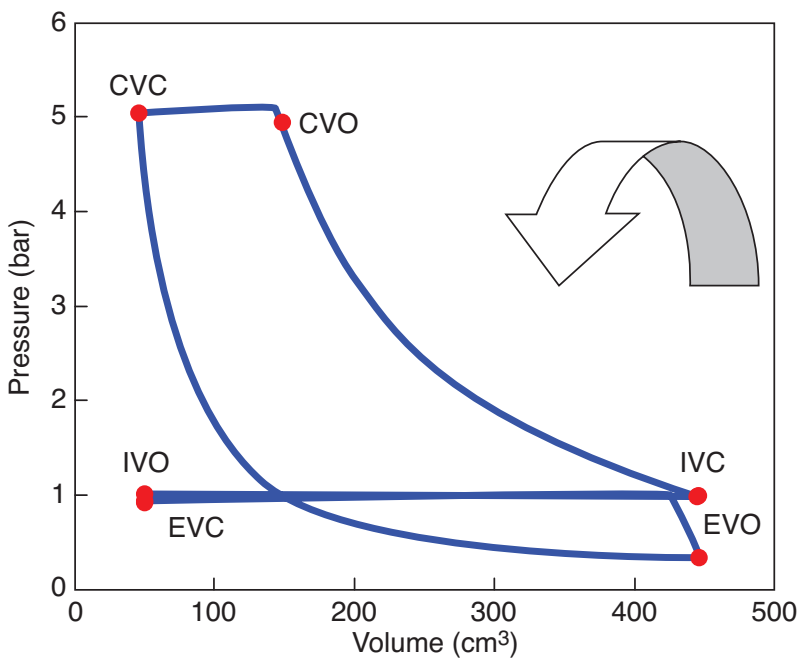

Figure 5

Pump mode indicator diagram.

CVC is the means to regulate a cycle work output. By delaying or advancing the CVC the total work output respectively grows or decreases. The exhaust stroke begins with Exhaust Valve Opening (EVO), followed by one expansion and compression phase. The rectification of the cams (intake and exhaust) is made in order to optimize the conventional mode, as this is the mode with the greatest influence on the consumption.

\subsubsection{Pump Mode}

The pumping mode (Fig. 5) is used to transform the kinetic energy from the vehicle to potential energy in the form of compressed air stored in the air-tank. Basically it is the opposite of the pneumatic mode (capturing air into the airtank instead of releasing it). The cycle begins with CVO during the compression stroke when the in-cylinder pressure reaches the air-tank pressure. The braking torque can then be adjusted by the timing of the CVC.

Further details about the thermodynamic cycles in the pneumatic-hybrid engine can be found in [4].

It is widely known that the combustion engine has lower efficiency at partial and poor load as shown in Figure 6. So, in this zone the pneumatic motor, as often as possible, will be used advantageously to cover the torque demands. In addition, even if the pneumatic motor performance (Fig. 7) is not always optimal it is important to remember that this energy is "free" because coming from recuperating mode. Another important advantage of the PHE concept is its downsizing potential as shown in [5], caused by the instantaneous availability of the compressed air to overcome turbocharger inertia. Since some energy management strategies are based on the relative efficiencies of the process, the modeling of propulsion modes became essential [6].

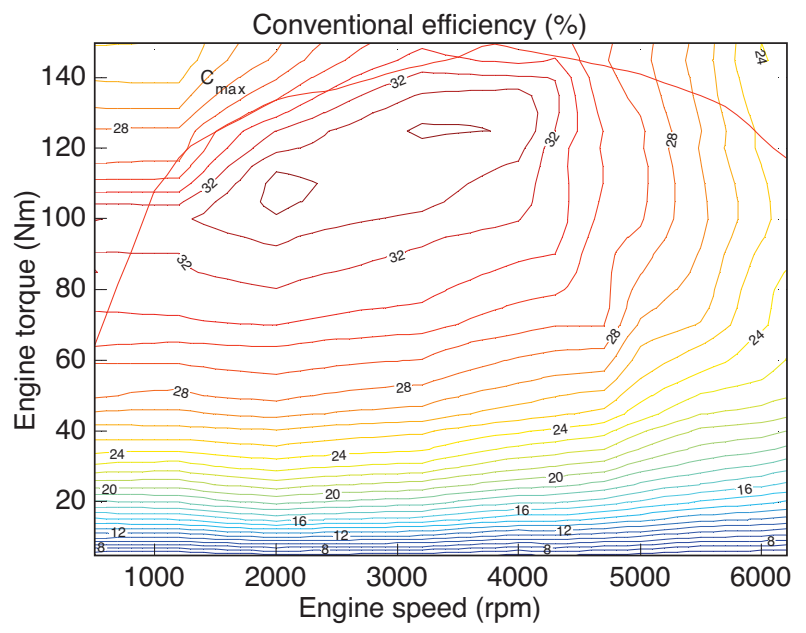

Figure 6

Conventional engine efficiency (\%) versus engine torque $(\mathrm{Nm})$ and speed (rpm).

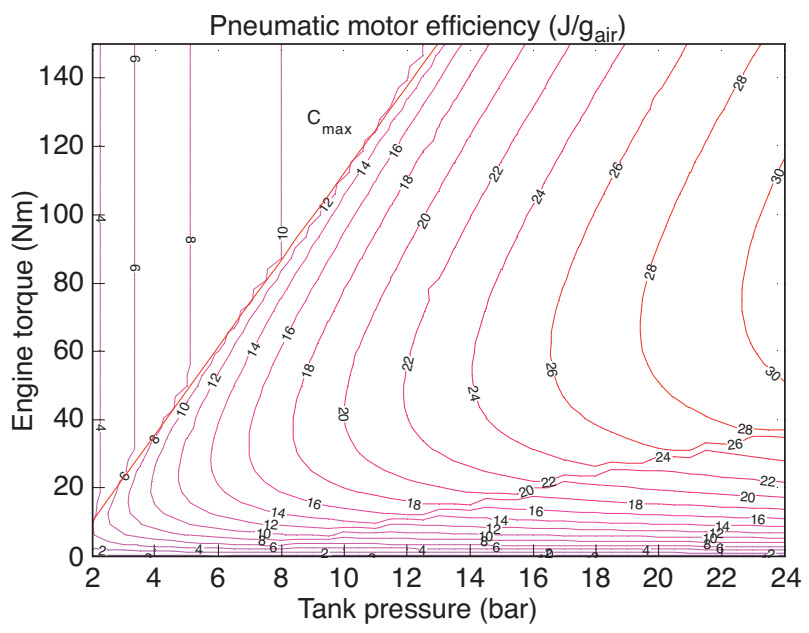

Figure 7

Pneumatic motor performance $\left(\mathrm{J} / \mathrm{g}_{\text {air }}\right)$ versus engine torque $(\mathrm{Nm})$ and tank pressure (bar).

\section{ENERGY MANAGEMENT STRATEGIES}

An energy management strategy can be considered as a decision system, in which the main objective is to minimize overall fuel consumption. The particularity of the PHE concept compared to other hybrid configurations dwells in the air-tank, which is only a short term storage device [7]. Energy management strategies can be divided into two main groups:

- online: in this case only information about the current status of the powertrain is required. Sometimes the history about the driven cycle or information from on-board sensors can be added to help adjust energy management performance; 
- offline: The knowledge of the overall cycle profile (from start to the end) yields the best possible energy savings. This knowledge is obviously impossible in a real environment, but it is used in dynamic programming to obtain the optimal solution. We can then benchmark the online energy management strategies.

A basic decision system, valid for all the energy management strategies, is also used as shown in Figure 8. If the torque is strictly negative, the chosen mode is the pneumatic pump (no dissipative vehicle braking), except when the deceleration torque is above the maximum of regenerative braking $T_{\text {pump_max }}$ where the classic brakes are engaged. If the demanded torque $T_{e}$ is equal to zero we can choose either to stop the engine (which assumes that the engine will restart at positive torque demand) or to leave it idling.

Finally if the demanded torque $T_{e}$ is positive, the strategies will choose the conventional or pneumatic mode.

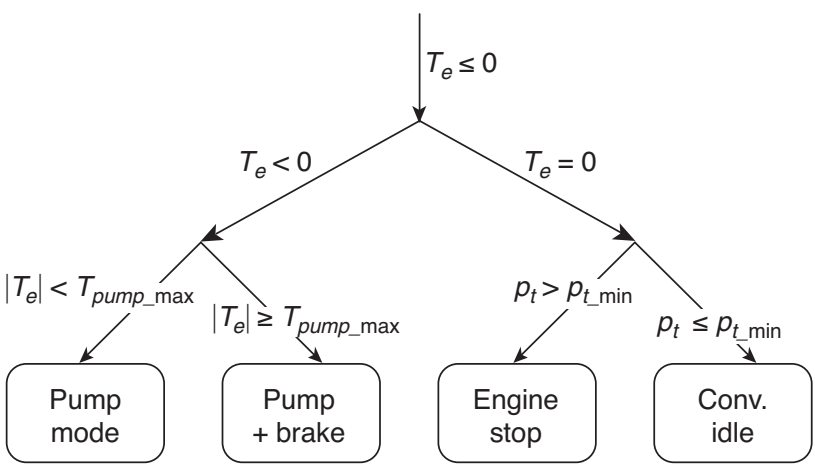

Figure 8

Basic decision tree valid for all strategies.

Four online strategies are compared here. First is the causal strategy in Section 2.1. Then in Section 2.2 two penalty coefficient strategies (constant and variable) are introduced. The last online strategy (in Sect. 3.3) is an improvement of two precedent strategies and uses a driving pattern recognition technique. After, all strategies are compared to the dynamic programming which is used as a reference (Sect. 3.4).

\subsection{Causal Strategy}

Causal Strategy (CS) implements a rule-based control technique. This strategy is strongly dependent on a specific application and it can give good results [8]. The system uses parameters such as engine speed $N_{e}$, demanded torque $T_{e}$ or tank pressure $p_{t}$ from which the decision of propulsion mode is made. In this paper, the pneumatic motor mode is used if there is enough tank pressure available; otherwise the conventional mode is chosen.

\subsection{Penalty Coefficient Strategy}

\subsubsection{Principle}

This strategy is based on the optimal control technique where minimizing the equivalent consumption between two energy sources. In certain conditions, both conventional and pneumatic propulsion sources can be used, but only one has to be chosen. The choice of the best mode is made in order to minimize the energy consumption defined in comparable units. In this case, consumption is compared in Joule as energy, which has to be taken from the fuel tank in the form of mass of gasoline $m_{f u e l}$ or from the air-tank as a mass of compressed air $m_{\text {air }}$. The lower equivalent consumption is chosen. Then the Penalty Coefficient (PC) is used as a charge sustaining factor which ensures that the air-tank pressure stays within lower and upper bounds. This is why we can also talk about Equivalent Consumption Minimization Strategy (ECMS) as described in $[9,10]$.

We define the energy to be taken from the different sources as:

$$
\begin{aligned}
& E_{\text {air }}=m_{\text {air }} \cdot C_{p} \cdot T_{t} \\
& E_{\text {fuel }}=m_{\text {fuel }} \cdot L C V
\end{aligned}
$$

where a Penalty Coefficient (PC) is used to balance consumption between fuel and compressed air. The equivalent energy factor is introduced into the objective function Equation (10) where the optimum value $J^{*}$ of energy to be consumed is found for each state during the simulation:

$$
J^{*}=\min \left\{E_{\text {air }} \cdot P C, E_{\text {fuel }}\right\}
$$

The Penalty Coefficient can be chosen Constant (CPC):

$$
P C=\lambda_{\text {mean }}
$$

or Variable (VPC) (Fig. 9), as a nonlinear function of the airtank pressure (by the definition of $x_{S O C}$ ):

$$
P C=\lambda_{\text {mean }}-x_{S O C}^{n}, \quad n>1
$$

In both cases the penalty coefficient function consists of a constant part $\lambda_{\text {mean }}$, and in the second case a function of a variable $x_{S O C}$, Equation (13) with the $n$-th power of the $x_{S O C}$ term was added, which expresses the tank pressure, thus prohibits entering the system boundaries.

$$
x_{S O C}\left(p_{t}\right)=\frac{2 \cdot p_{t}-\left(p_{t_{-} \max }+p_{t_{-} \min }\right)}{p_{t_{-} \max }-p_{t_{-} \min }}
$$

The choice of the value of $\lambda_{\text {mean }}$ is very important. If it is too small, it will lead to a very fast and "non economic" discharge of the air-tank. On the other hand too high value 


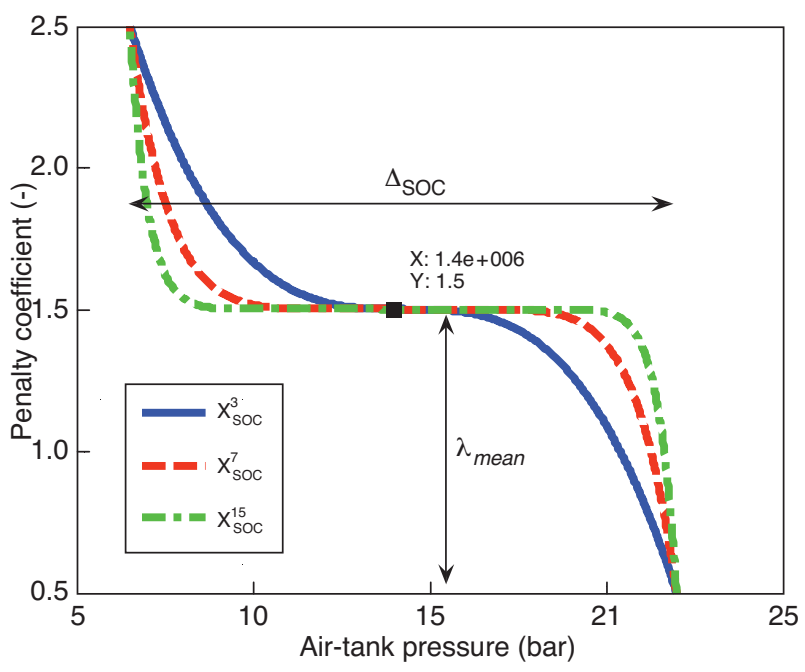

Figure 9

Non-linear function of variable penalty coefficient.

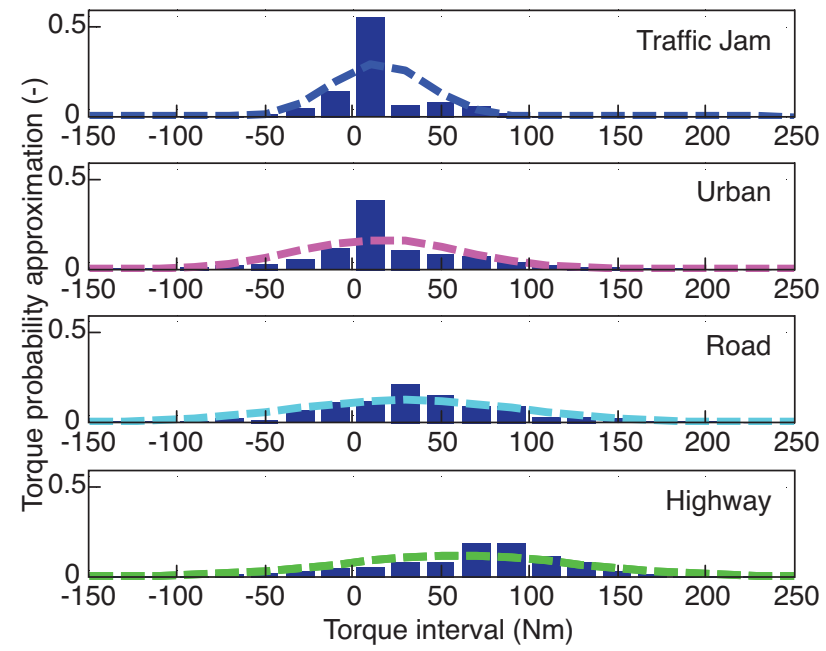

Figure 10

Patterns as probability density of Artemis driving cycles. would lead to not using the pneumatic energy, thus removing the advantage of hybrid propulsion. In the VPC case the $x_{S O C}$ introduce the boundaries $p_{t \_ \text {min }}$ and $p_{t_{-} \max }$ which permits to obtain an additional progressive sensibility in order to maintain the air-tank pressure inside the limit values. The results obtained by both strategies CPC and VPC are compared to gain insight into their functionality and further possibilities for improvement. For example the influence of the additional parameter $n$ which can reshape the non linear function as show in Figure 9 will be studied in Section 3.1.

\subsubsection{Computing the Penalty Coefficients}

The optimal penalty coefficients are computed during the off-line optimization. Since the air-tank is considered as a short term storage system, the assumption which holds for a hybrid electric system of neglecting the variation in the battery internal parameter cannot be used. Then two types of the optimal penalty coefficients have been established which attains the best consumption results:

- for each of the driving situation separately - local;

- over the family of identified driving situations - global.

\subsection{Driving Pattern Recognition}

\subsubsection{Principle}

This strategy is based on the assumption that the current driving situation does not change rapidly and thus is likely to continue into the near future. In this strategy a pattern recognition technique is used to adapt the penalty coefficient during the driving cycle. In the literature, different methods have been used to describe and recognize the driving situation $[11,12]$.
A pattern is a model which can be used to recognize a specific driving condition. We propose to define the patterns as a statistical model and the pattern recognition method as a correlation function. The sliding window size, in which the pattern identification is done, has to be adjusted to optimize performance.

\subsubsection{Statistical Model for the Pattern}

Two statistical evaluation techniques were used to characterize the driving conditions. The first technique is the density of probability, where the normal distribution was assumed Equation (14). The second is the density of presence (as histograms). In both cases (Fig. 10) the torque demand $T_{e} i$ s used as the main identification parameter and the mean value of the vehicle speed is also associated to the probabilistic pattern model.

$$
\phi_{\mu, \sigma^{2}}(x)=\frac{1}{\sigma \sqrt{2 \pi}} \cdot \exp \left(-\frac{(x-\mu)^{2}}{2 \sigma^{2}}\right),
$$

where $\sigma^{2}$ is the variance and $\mu$ is the mean value of the torque $T_{e}$.

\subsubsection{Pattern Recognition}

From the identified driving situations, four reference patterns are used in the driving pattern recognition strategy (trafficjam, urban, road and highway).

In order to recognize a driving situation, correlation between actual and reference pattern is computed on a sliding window. The greatest correlation coefficient gives the identified pattern. In the case when the correlation coefficients are relatively close to each other, the mean speed values help to 
distinguish the reference patterns from each other. Then the value of penalty coefficient is set and hence adapted according to the recognized pattern.

The identification window slides along the driving cycle with the sliding period of one simulation step. The set of different window sizes from a few seconds to several minutes was examined. Then the optimal window size was selected according to the success of identification on the reference cycle (see the result part).

\subsection{Dynamic Programming}

\subsubsection{Principle}

Knowing the future profile of the driving cycle and having a precise model of the system, we can calculate all the possible states during the driving cycle using the Bellman principle of optimality. This allows us to find the sequence of activated modes with the smallest overall consumption.

The forward Dynamic Programming (DP), which is equivalent to a backward dynamic programming [13], is used as a reference. In same conditions (initial and final states) a perfect comparison between online strategies and DP can be done. In this case DP gives the optimal cost to reach.

Figure 11 shows the principle of forward DP. At the beginning $(t=0 \mathrm{~s})$ the initial tank pressure is $p_{0,1}=10 \mathrm{bar}$. At each time step, a torque demand $T_{e}$ has to be satisfied. If $T_{e}$ is positive, two propulsion modes can be chosen:

- conventional mode: the fuel mass necessary to produce the desired torque is consumed but at the same time the compressed air is not used;

- pneumatic mode: no fuel mass is consumed. Only the necessary air mass is taken from the air-tank to produce the desired torque. Then the tank pressure falls to value $p_{1,2}$.

This procedure is reiterated considering all other modes until the end of the driving cycle.

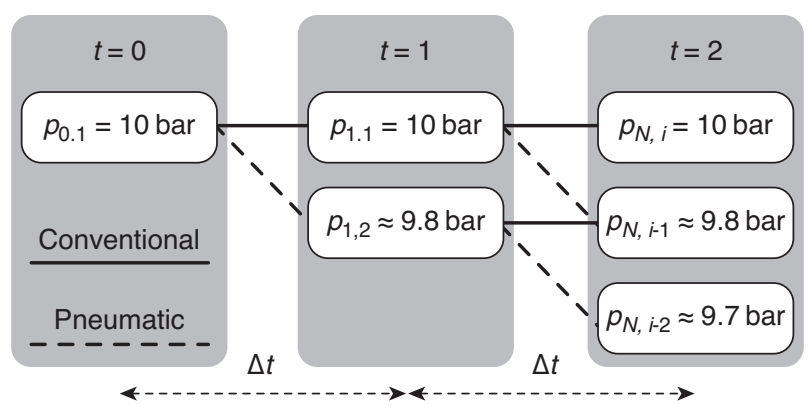

Figure 11

Principle of forward dynamic programming.

\subsubsection{Mathematical Approach}

A discrete-time dynamic system is considered:

$$
x_{k+1}=f_{k}\left(x_{k}, u_{k}\right), \quad k=0,1, \ldots, N-1
$$

where $x_{k}$ is an element of a space $S_{k}$ (vehicle speed, demanded torque and tank pressure), here $x_{k}$ is the tank pressure. Since the driving cycle is known in advance the vehicle speed $v_{d}$ and torque demand $T_{e}$ are imposed. Control $u_{k}$ is an element of a space $C_{k}$ and is regarded as a space of propulsion modes of the hybrid drive powertrain. The control $u_{k}$ is constrained to take values in a given nonempty subset $U\left(x_{k}\right) \subset C_{k}$, of modeled propulsion modes which depends on the current state $x_{k}$; that is, $u_{k} \in U_{k}\left(x_{k}\right), \forall x_{k} \in S_{k}$ and $k$.

We consider the class of policies $\pi$ (also called control laws or global strategy) that consists of a sequence of functions:

$$
\pi=\left\{\mu_{0}, \ldots, \mu_{N-1}\right\}
$$

where $\mu_{k}$ maps states $x_{k}$ into controls $u_{k}=\mu_{k}\left(x_{k}\right)$ and is such that $\mu_{k}\left(x_{k}\right) \in U_{k}\left(x_{k}\right)$ for all $x_{k} \in S_{k}$. Such policies will be called admissible or chosen modes.

Given an initial state $x_{0}$ and an admissible policy $\pi$, the states are variables with distribution defined through the system equation.

$$
x_{k+1}=f\left(x_{k}, \mu_{k}\left(x_{k}\right)\right)
$$

Thus for a given cost function (here only a fuel consumption) $g_{k}, k=0,1, \ldots, N-1$ the expected trajectory cost of $\pi$ sequence of modes starting at $x_{0}$ is:

$$
J_{\pi}\left(x_{0}\right)=E\left\{g_{N}\left(x_{N}\right)+\sum_{k=0}^{N-1} g_{k}\left(x_{k}, \mu_{k}\left(x_{k}\right)\right)\right\}
$$

where the expectation cost is taken over the variable $x_{k}$. An optimal policy $\pi^{*}$ is one that minimizes this cost;

$$
J_{\pi^{*}}\left(x_{0}\right)=\min _{\pi \in \pi} J_{\pi}\left(x_{0}\right)
$$

where $\pi$ is the set of all admissible sequences.

We should not forget that the optimal policy $\pi^{*}$ is associated with a fixed initial state $x_{0}$. However, an interesting aspect of dynamic programming is that in particular cases it is possible to find a policy $\pi^{*}$ that is simultaneously optimal for all initial states [13].

The optimal cost depends on $x_{0}$ and is denoted by $J^{*}\left(x_{0}\right)$; that is:

$$
J^{*}\left(x_{0}\right)=\min _{\pi \in \pi} J_{\pi}\left(x_{0}\right)
$$

It is useful to view $J^{*}$ as a function that assigns to each initial state $x_{0}$ the optimal cost $J^{*}\left(x_{0}\right)$ and call it the best possible consumption or optimal value.

\section{RESULTS}

Results are divided into three parts. We first discuss the influence of different shapes of the penalty coefficient function on the 
final consumption, where the pattern recognition can play an advantageous role if we consider the fuel consumption differences obtained by global and local $\lambda_{\text {mean }}$ values. The optimization of the sliding window size for pattern recognition is then presented. Third and last result part is devoted to maximum attainable gain obtained by the DP, where the knowledge of the future driving cycle is mandatory. We conclude with a summarizing table in which the final consumptions for different energy management strategies are presented on two cycles. The initial tank pressure is always 10 bar.

\subsection{Influence of the Shape of Penalty the Function}

Three different shapes of the penalty coefficient function are compared on the bases of fuel consumption during the reference cycles. Two summarizing tables show results for different PC shapes.

- Table 1: Constant Penalty Coefficient (CPC);

- Table 2: Variable Penalty Coefficient (VPC), where $x_{S O C}$ have lower and higher $n$.

Table 1 is divided into two subparts, where the potential of the pattern recognition can be observed:

- an upper part for the penalty coefficient optimized locally for each of the reference cycle;

- a lower part for the penalty coefficient optimized globally over all of the reference patterns.

The PC is constant, which means that the balance between energy consumption of pneumatic and chemical energy is kept the same throughout the driving cycle. This strategy is the simplest approach but does not ensure the availability of the charge $x_{S O C}$ in the air-tank throughout the driving cycle. On the other hand the consumption gain benefit of the pattern recognition can be clearly seen if we compare two parts of the table.

In Table 2, we vary $n$ of Equation (12) and see the influence of the $\lambda_{\text {mean }}$ tuning (local for one driving cycle or global for all driving cycles) on fuel consumption.

Table 2 is divided into four subparts, where the PC shape influence can be seen separately for each of driving situation.

TABLE 1

Constant Penalty Coefficient (CPC)

\begin{tabular}{c|c|c|c|c}
\hline Cycle & Traffic-jam & Urban & Road & Highway \\
\hline$\lambda_{\text {mean }}$ local & 2.05 & 1.5 & 1.35 & 1.3 \\
\hline Cons. $(\mathrm{L} / 100 \mathrm{~km})$ & 8.54 & 6.81 & 5.51 & 8.72 \\
\hline$p_{t \text {-final }}$ & 11.73 & 8.33 & 9.97 & 10.25 \\
\hline$\lambda_{\text {mean }}$ global & \multicolumn{5}{|c}{1.55} \\
\hline Cons. $(\mathrm{L} / 100 \mathrm{~km})$ & 9.42 & 6.82 & 5.75 & 8.73 \\
\hline$p_{t \text {-final }}$ & 6.94 & 8.4 & 21.43 & 20.94 \\
\hline
\end{tabular}

The first column is the best fuel consumption with lower $n$ local $\lambda_{\text {mean }}$ optimization. The second column is also with lower $n$ but this time the $\lambda_{\text {mean }}$ is optimized globally over all driving situations. The third column manifests the benefit of the higher $n$. with local optimization. In the fourth column $n$ and $\lambda_{\text {mean }}$ have been tune to obtain the global minimal fuel consumption.

Several observations can be made:

- difference in the global and local tuning is significant, thus promising for pattern recognition;

- higher $n$ in the SOC term was found useful only during the traffic-jam cycle. This is due to the cycle nature, where the use of pneumatic energy is the most beneficial;

- the best global consumption for VPC strategy is with the higher $n$.

Another interesting point is the influence of changing the $\mathrm{PC}$ on the cycle consumption, which is biggest on the trafficjam cycle. Therefore we can expect major improvements with the DPR strategy.

TABLE 2

Variable Penalty Coefficient (VPC)

\begin{tabular}{|c|c|c|c|c|}
\hline \multirow{2}{*}{$\begin{array}{c}\text { Cycle } \\
\text { optimized }\end{array}$} & \multicolumn{4}{|c|}{ Traffic-jam } \\
\hline & Local & Global & Local & Global \\
\hline$n$ & 3 & 3 & 15 & 11 \\
\hline$\lambda_{\text {mean }}$ & 1.5 & 1.35 & 1.6 & 1.5 \\
\hline Cons. (L/100 km) & 8.25 & 8.35 & 8.09 & 8.23 \\
\hline$p_{t \text {-final }}($ bar $)$ & 11.12 & 9.63 & 9.26 & 8.67 \\
\hline \multirow{2}{*}{$\begin{array}{c}\text { Cycle } \\
\text { optimized }\end{array}$} & \multicolumn{4}{|c|}{ Urban } \\
\hline & Local & Global & Local & Global \\
\hline$n$ & 3 & 3 & 15 & 11 \\
\hline$\lambda_{\text {mean }}$ & 0.75 & 1.35 & 1.4 & 1.5 \\
\hline Cons. (L/100 km) & 6.91 & 7.04 & 6.99 & 6.97 \\
\hline$p_{t \text {-final }}(\mathrm{bar})$ & 7.91 & 9.85 & 9.05 & 9.52 \\
\hline \multirow{2}{*}{$\begin{array}{c}\text { Cycle } \\
\text { optimized }\end{array}$} & \multicolumn{4}{|c|}{ Road } \\
\hline & Local & Global & Local & Global \\
\hline$n$ & 3 & 3 & 15 & 11 \\
\hline$\lambda_{\text {mean }}$ & 0.45 & 1.35 & 0.2 & 1.5 \\
\hline Cons. (L/100 km) & 5.53 & 5.66 & 5.54 & 5.77 \\
\hline$p_{t \text {-final }}(\mathrm{bar})$ & 9.26 & 12.64 & 8.7 & 20.7 \\
\hline \multirow{2}{*}{$\begin{array}{c}\text { Cycle } \\
\text { optimized }\end{array}$} & \multicolumn{4}{|c|}{ Highway } \\
\hline & Local & Global & Local & Global \\
\hline$n$ & 3 & 3 & 15 & 11 \\
\hline$\lambda_{\text {mean }}$ & 0.45 & 1.35 & 1.45 & 1.5 \\
\hline Cons. (L/100 km) & 8.73 & 8.75 & 8.73 & 8.75 \\
\hline$p_{t \text {-final }}($ bar $)$ & 7.72 & 12.43 & 13.18 & 13.4 \\
\hline
\end{tabular}




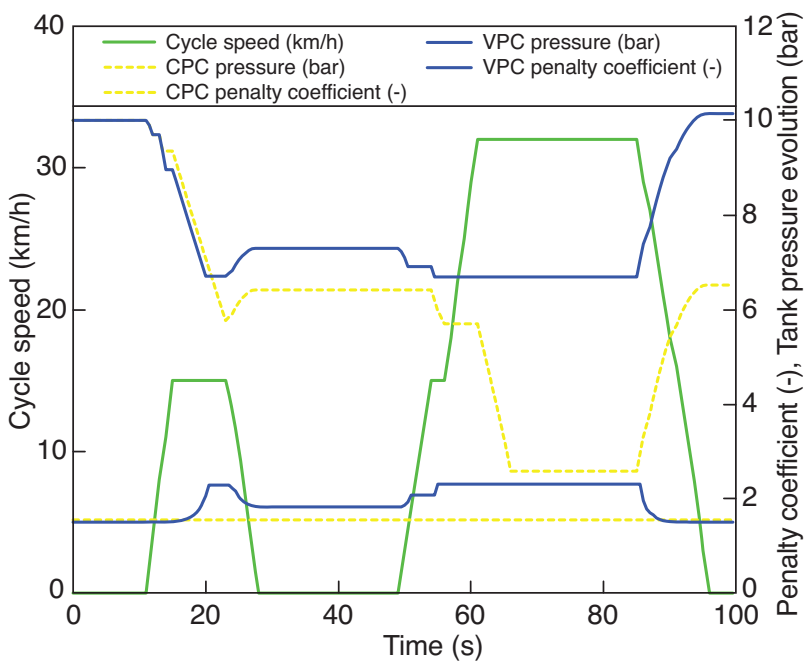

Figure 12

Evolution of the state of the charge CPC and VPC at beginning of the NEDC driving cycle.

If we summarize the results for different PC function shapes: CPC is the simplest approach, but the disadvantage is that the pressure in the air-tank can easily reach the limit values, thus not to use the pneumatic Stop\&Start function. The VPC strategy erases this disadvantage. The tank pressure evolution comparing the CPC and VPC strategy can be seen in Figure 12. In particular between 30 and $50 \mathrm{~s}$ with CPC the engine is kept idling because not enough pressure $\left(p_{t}<p_{t \_ \text {min }}\right)$ is available to restart the engine (at $50 \mathrm{~s}$ ). In the opposite with VPC enough pressure has been maintained to restart the engine.

The benefit of reshaping the VPC function with higher $n$ has been observed only during the Traffic Jam cycle. In all cases the pattern identification technique can provide a significant advantage in terms fuel saving because of appropriate tuning of the penalty coefficient.

\subsection{The Optimum Size for the Sliding Window}

The optimum identification window size was established by varying the length of the sliding window on the reference cycle. The best recognition success was attained with a 3-minute-long window for the density of presence and five minutes for the probability density evaluation technique, Equation (14). The results of this study are shown in Figure 13.

The evaluation with a density of presence was further used (Fig. 14) because of slightly better identification results and smaller window size.

\subsection{Dynamic Programming Results}

As can be seen in Table 3 the achievable gains are very high for the traffic-jam cycle, where the hybrid potential can be

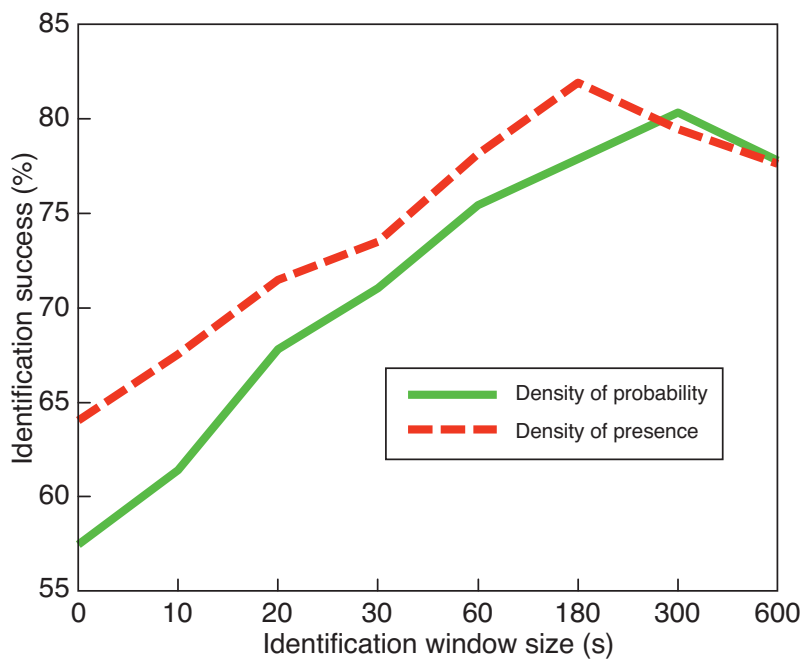

Figure 13

Identification success as a function of the window size on the reference cycle.

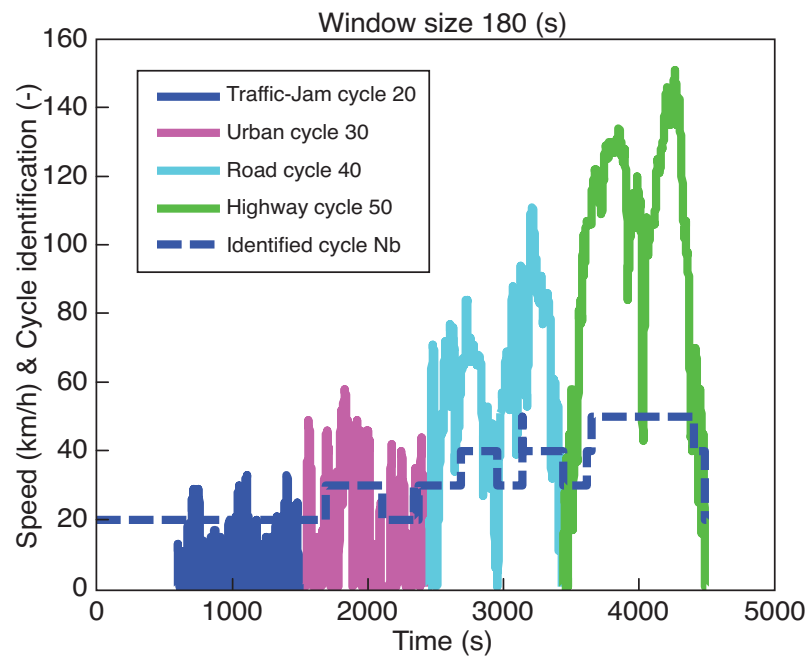

Figure 14

Reference cycle recognition.

mainly exploited. As we approach urban and extra urban driving conditions, the hybridation gains diminish. This is due to the efficiency characteristics, where the conventional engine has the best operation points and has no problem with charge sustainability.

It should be mentioned that these gains are the optimal ones and thus to achieve them, perfect knowledge of the driving cycle is mandatory. This strategy is therefore used as a reference for benchmark and is not directly applicable to a real driving vehicle. 
TABLE 3

Achievable gain with the optimal DP solution (offline)

\begin{tabular}{c|c|c|c|c}
\hline Cycle/strategy & Traffic-jam & Urban & Road & Highway \\
\hline Conventional & 14.41 & 10.09 & 6.13 & 8.89 \\
\hline$g_{\mathrm{CO}_{2}} / \mathrm{km}$ & 346 & 242 & 147 & 213 \\
\hline $\mathrm{DP}$ & 7.55 & 6.75 & 5.5 & 8.7 \\
\hline$g_{\mathrm{CO}_{2}} / \mathrm{km}$ & 181 & 162 & 132 & 209 \\
\hline Achievable gain & $48 \%$ & $33 \%$ & $10 \%$ & $2 \%$ \\
\hline$p_{t \text {-initial }}$ & \multicolumn{5}{|c}{10} \\
\hline$p_{t \text {-final }}$ & \multicolumn{5}{|c}{10} \\
\hline
\end{tabular}

\subsection{Energy Management Strategies}

The results for different driving cycles and energy management strategies are summarized in Table 4. It is shown that the strategies based on pattern recognition are the ones with the lowest fuel consumption. One exception is in the NEDC cycle. This is due to identification delay and also to the fact that the NEDC cycle is composed of four urban (3 minutes long) and one extra urban (5 minutes long) patterns, which are proportionally short compared to the 3 minutes window size need to be considered. In all cases the values of penalty coefficients were used according to the optimal ones found in previous tables.

TABLE 4

Energy management strategies results

\begin{tabular}{c|c|c|c|c|c|c}
\hline \multirow{2}{*}{$\begin{array}{c}p_{t \text {-initial }} \\
\text { all 10 bar }\end{array}$} & \multicolumn{3}{|c|}{$\mathrm{NEDC}$} & \multicolumn{3}{c}{ Reference cycle } \\
\cline { 2 - 7 } & Cons. & $\mathrm{CO}_{2}$ & $p_{t \text {-final }}$ & Cons. & $\mathrm{CO}_{2}$ & $p_{t \text {-final }}$ \\
\hline Strategy & $\mathrm{L} / 100 \mathrm{~km}$ & $\mathrm{~g} / \mathrm{km}$ & bar & $\mathrm{L} / 100 \mathrm{~km}$ & $\mathrm{~g} / \mathrm{km}$ & bar \\
\hline Conventional & 7.2 & 173 & - & 8.2 & 197 & - \\
\hline Causal & 6.15 & 148 & 20.26 & 7.73 & 186 & 23.36 \\
\hline CPC & 6.08 & 146 & 18.24 & 7.6 & 182 & 23.23 \\
\hline CPC+DPR & 6.15 & 148 & 17.98 & 7.35 & 176 & 10.23 \\
\hline VPC & 6.06 & 145 & 19.29 & 7.53 & 181 & 19.98 \\
\hline VPC+DPR & 6.07 & 146 & 18.07 & 7.33 & 176 & 8.27 \\
\hline DP & 5.93 & 142 & 19.29 & 7.24 & 174 & 8.27 \\
\hline
\end{tabular}

We can conclude that the best results are attained with the VPC+DPR strategy even if the pattern recognition is not instantaneous as shown in Figure 14. This figure shows that the optimized values of PC are not used immediately, but with a short delay. Two solutions are conceivable, the simpler solution with CPC and DPR strategy and a more complex VPC also with DPR strategy. The final SOC for the DP solution was chosen to match the best of the online strategies. For comparative reasons, the final SOC difference for other strategies was neglected. The fuel consumption is expressed in liters per $100 \mathrm{~km}$ and $\mathrm{CO}_{2}$ emissions are recalculated with the equivalence of $2.4 \mathrm{~kg} \mathrm{CO}_{2}$ per liter of fuel.

\section{CONCLUSION}

We have provided a scale of the applicable energy management strategies. The focus was made on the penalty coefficient strategy, where the optimal values of penalty coefficients were established on reference patterns. These values were then used during the pattern recognition process. The driving pattern recognition benefit was confirmed on several driving cycles. The fuel consumptions were also compared to the best attainable obtained by Dynamic Programming, where the close to optimal results were observed.

For further work the random cycle development technique can help to validate the proposed strategies [14] and the pattern recognition can be improved to obtain a prediction capacity, in which fewer assumptions need to be made.

\section{REFERENCES}

1 Higelin P., Charlet A., Chamaillard Y. (2002) Thermodynamic Simulation of a Hybrid Pneumatic-Combustion Engine Concept, Int.J. Appl. Thermodynam. 5, 1, 1-11.

2 Schechter M.M. (1999) New Cycles for Automobile Engines, in International Congress and Exposition, Detroit, Michigan, 199901-0623.

3 Andre M. (2004) The ARTEMIS European driving cycles for measuring car pollutant emissions, Sci. Total Environ. 73-84, 334-335.

4 Brejaud P. et al. (2008) Pneumatic-Combustion Hybrid Engine: A study of the effect of the valvetrain sophistication on pneumatic modes, in Advances in Hybrid Powertrains, Paris.

5 Donitz C. et al. (2009) Realizing a concept for high efficiency and excellent driveability: The downsized and supercharged hybrid pneumatic engine, in SAE World Congress 2009, Detroit, USA 2009-01-1326.

6 Donitz C. et al. (2009) Modelling and optimizing two- and fourstroke hybrid pneumatic engines, IMechE, Part D: J. Automobile Eng. 223, 2, 199-209.

7 Guzzella L., Sciarretta A. (2007) Vehicle Propulsion Systems. Introduction to Modeling and Optimization, Second Edition, Springer.

8 Scirretta A., Guzzella L. (2003) Rule-based and optimal control strategies for energy management in parallel hybrid vehicles, in 6th International Conference on Engines for Automobile 2003, Capri, SAE_NA 2003-01-32.

9 Rizzonil G., Pisu P., Calo E. (2003) Control strategies for parallel hybrid electric vehicles, in Advanced in automotive control 2003, Salerno, Italy.

10 Paganelli G. et al. (2002) Equivalent consumption minimization strategy for parallel hybrid powertrains, in IEEE Vehicular Technology Conference 2002. 
11 Jeon S.-I. et al. (2002) Multi-Mode Driving Control of a Parallel Hybrid Electric Vehicle Using Driving Pattern Recognition, ASME 124, 1, 141-149.

12.Lin C.-C. et al. (2002) Control of a Hybrid Electric Truck Based on Driving Pattern Recognition, in Advanced Vehicle Control Conference 2002, Hiroshima, Japan.

13 Bertsekas D.P. (2005) Dynamic Programming and Optimal Control, 3rd ed., Vol. 1, Nashua, USA, Athena Scientific, 540.
14 Ivanco A. et al. (2009) Energy management strategies for hybrid-pneumatic engine studied on an Markov Chain type generated driving cycle, in SAE World Congress 2009, Detroit, Michigan, 2009-01-0145.

Final manuscript received in July 2009 Published online in November 2009 